\title{
Characterization of chicken riboflavin carrier protein gene structure and promoter regulation by estrogen
}

\author{
NANDini VASUdeVAN ${ }^{\dagger}$, URVASHi BAHAduR and PATURU KONDAiAH* \\ Department of Molecular Reproduction, Development and Genetics, Indian Institute of Science, \\ Bangalore 560 012, India \\ ${ }^{\dagger}$ Present address: Laboratory of Neurobiology and Behavior, Rockefeller University, 1230, York Avenue, \\ New York, NY 10021, USA \\ *Corresponding author (Fax, 91-80-360 0999; Email, paturu@serc.iisc.ernet.in).
}

\begin{abstract}
The chicken riboflavin carrier protein (RCP) is an estrogen induced egg yolk and white protein. Eggs from hens which have a splice mutation in RCP gene fail to hatch, indicating an absolute requirement of RCP for the transport of riboflavin to the oocyte. In order to understand the mechanism of regulation of this gene by estrogen, the chicken RCP gene including $1 \mathrm{~kb}$ of the $5^{\prime}$ flanking region has been isolated. Characterization of the gene structure shows that it contains six exons and five introns, including an intron in the $5^{\prime}$ untranslated region. Sequence analysis of the $5^{\prime}$ flanking region does not show the presence of any classical, palindromic estrogen response element (ERE). However, there are six half site ERE consensus elements. Four deletion constructs of the $5^{\prime}$ flanking region with varying number of ERE half sites were made in pGL3 basic vector upstream of the luciferase-coding region. Transient transfection of these RCP promoter deletion constructs into a chicken hepatoma cell line (LMH2A) showed 6-12-fold transcriptional induction by a stable estrogen analogue, moxesterol. This suggests that the RCP gene is induced by estrogen even in the absence of a classical ERE and the half sites of ERE in this promoter may be important for estrogen induction.
\end{abstract}

\section{Introduction}

The chicken riboflavin carrier protein (RCP) belongs to a class of vitamin carrier proteins that bind vitamins reversibly and with high affinity. RCP is a phosphoglycoprotein present in egg yolk and egg white (reviewed in Adiga 1994). Egg white RCP contains 219 amino acids with nine disulphide bonds (Hamazume et al 1984, 1987). The egg yolk RCP is identical to the egg white RCP but for the deletion of 11-13 amino acids at the $\mathrm{C}$ terminus due to limited proteolytic cleavage during oocyte uptake (Adiga 1994). The chicken and turtle RCP cDNAs have been cloned and the deduced amino acid sequences show $71 \%$ similarity (Zheng et al 1988; Hamajima and Ono 1995).
Eggs from hens which have a $3^{\prime}$ splice site mutation in the RCP gene fail to hatch, demonstrating an absolute necessity of RCP protein for embryonic development (White and Merrill 1988; MacLachlan et al 1993). Embryonic development can be rescued by the administration of riboflavin into these eggs, suggesting that the principal function of RCP is the transport of the vitamin to the oocyte. Active immunization of mammals using chicken RCP resulted in termination of pregnancy, pointing to a vital role for an RCP-like molecule in the maintenance of pregnancy (reviewed in Adiga 1994).

In oviparous species, egg yolk and egg white proteins are synthesized in the liver and oviduct respectively under the influence of estrogen. For the synthesis of chicken RCP in both the liver and oviduct, estrogen

Keywords. ERE half site; gene expression; liver

Abbreviations used: RCP, Riboflavin carrier protein; FBS, fetal bovine serum; ERE, estrogen response element; PCR, polymerase chain reaction; PRE, progesterone response element. 
is essential. RCP levels in the sera of immature male birds can be detected following a single 17- $\beta$ estradiol administration after $6 \mathrm{~h}$ and reach a peak at $48 \mathrm{~h}$ (Murthy and Adiga 1978).

Among estrogen regulated genes, RCP and conalbumin are the only genes expressed in both liver and oviduct. However, unlike conalbumin which is upregulated by estrogen in the liver, RCP gene regulation is strictly dependent on the presence of estrogen in both liver and oviduct. Hence it provides a better model to study the tissue and hormone specific induction of gene expression by estrogen. In order to elucidate the mechanism(s) of chicken RCP gene regulation, the structure and the 5' flanking region of the gene have been characterized.

\section{Methods}

\subsection{Isolation of RCP genomic clones}

A chicken genomic library in lambda Charon 4A vector was screened with chicken RCP cDNA (Zheng et al 1988) labelled with $\left[\alpha-{ }^{32} \mathrm{P}\right] \mathrm{dCTP}(3000 \mathrm{Ci} / \mathrm{mmol}$, NEN Research Products, USA) as per standard protocols (Sambrook et al 1989). The inserts from the positive clones hybridizing to the RCP cDNA were subcloned into pBluescript and pTZ18R plasmid vectors and subjected to automated sequencing (ABI Inc., USA).

\subsection{Transcription start site mapping}

Transcription start site mapping was carried out using the primer extension method. Briefly, two overlapping antisense oligonucleotides, $29 \mathrm{PE}$ and $99 \mathrm{PE}$ (figure 1 for locations of the primers) were labelled using $\left[\gamma_{-}{ }^{32} \mathrm{P}\right] \mathrm{ATP}$ (NEN Life Science Products, USA) and were extended using Superscript II reverse transcriptase (Gibco BRL Products, Life Technologies, USA) on $50-100 \mu \mathrm{g}$ of hen liver total RNA. The reverse transcription products were resolved on a $5 \%$ sequencing gel containing $7 \mathrm{M}$ urea and exposed to an X-ray film (Ausubel et al 1995). An $\left[\alpha-{ }^{32} \mathrm{P}\right]$-labelled, pBR322 HinfI ladder and a sequencing ladder were used to size the extension products obtained by using the two primers.

\subsection{RNA extraction and Northern blot analysis}

Total RNA from cells was isolated by the method of Chomczynski and Sacchi (1987). The RNA was resolved on a $1.2 \%$ agarose gel containing $2.2 \mathrm{M}$ formaldehyde in MOPS buffer as described (Sambrook et al 1989). The RNA was blotted onto Hybond membrane (Amersham Pharmacia PLC Inc., UK) and hybridized to $\left[\alpha-{ }^{32} \mathrm{P}\right] \mathrm{dCTP}$ labelled RCP cDNA (Zheng et al 1988) and chicken glyceraldehyde phosphate dehydrogenase (GAPDH) probes. Hybridization and washing of the blots were carried out as described by Church and Gilbert (1984) and they were exposed to X-ray films.

\subsection{Promoter reporter constructs of the $5^{\prime}$ flanking region of $R C P$ gene}

The PCR products amplified using various primer sets spanning the RCP $5^{\prime}$ flanking region and a vector primer (for the longest construct) are subcloned into the pGL3 basic vector (Promega Corpn., USA) to generate progressive deletions in the $5^{\prime}$ flanking region, designated as pRCPluc 1, 2, 3 and 4. The details of the position of the deletions are indicated in figure 4A. The forward primer sequences are:

(i) AATTAACCCTCACTAAAGGG,

(T3 primer, pBluescript for pRCPluc4),

(ii) CGGGGtACCGCTTTCTGTGATGGTCCATG, (pRCPluc3),

(iii) GTCGCTAGCTTAACCCAAAGCAAACTG, (pRCPluc2),

(iv) CGGGAGCTCTTTTCTAATCCTAGTCTG,

(pRCPluc1) and the reverse primer was

TGCAGATCTTCCTTCTGGGTGAGAGTTC.

The plasmid DNA for transfection was prepared using plasmid isolation columns (Qiagen $\mathrm{GmbH}$, Germany).

\subsection{DNA transfections and reporter assays}

Chicken hepatoma cells (LMH2A, ATCC CRL-2118) were grown in tissue culture plates coated with $1 \%$ gelatin using Waymouth's Medium (Gibco BRL Life Technologies, USA) supplemented with $10 \%$ fetal bovine serum (FBS, Sigma Aldrich, USA) in a $5 \% \mathrm{CO}_{2}$ humidified atmosphere. For transfection of DNA, cells were seeded in 6-well clusters (Nunc products, Germany) at a density of $2 \times 10^{5}$ per well and allowed to attach and grow for $48 \mathrm{~h}$. Transfections were carried out in phenol red free DMEM (Gibco-BRL Life Technologies, USA) using $3 \mu \mathrm{g}$ of Lipofectin reagent (Gibco-BRL Life Technologies, USA), $2 \mu \mathrm{g}$ of each deletion construct and $50 \mathrm{ng}$ of pRL-TK plasmid (Promega Corpn., USA), according to the manufacturers' protocol. pRL-TK is a plasmid encoding renilla luciferase used for normalization of transfection efficiencies and cell lysis. After $6 \mathrm{~h}$ of transfection, fresh phenol red free DMEM containing 10\% charcoal stripped FBS was added and the cells were allowed to recover. After $24 \mathrm{~h}$ of recovery, cells were treated with $50 \mathrm{nM}$ moxesterol (NEN life science products, USA) dissolved in ethanol, or with vehicle. The cells were lysed after $12 \mathrm{~h}$ 
NF1

NF1 NF1 GRE

Pit1 ERE

-973 GATCCATTATCATACTGCGAGTTTGCTCAGTGAGATTCATTCCACACTTCCACTGTGCCAGTGTTTGTTTTATTCAAGCAAAAAAGTTITGTAAATACTGACCCACAGTTAC GRE NF1, OCt1 AP3

-861 TATTTGACAAACCACTGTTGTGTTTTAAAATAGAAACAAGAGATGCTATTTTCCATTTGCATCTGAATAATTGCAAAGTAGTCAGTGGCGTGTTGCTAGTTTGGGAGCTCAC ERE ERE

-749 TGGGATTIGACCTATGGAAGTAAGTGCAGGTATTTGTAATGACCACGTCTGCTTTCTGTGATGGTCCATGTTCAGATATGGAATCCCCTCTGCAGAAAGCACACCTTGTAAG PEA-3.NF1

-637 GAAATCCAGTCAGCAACTGCTGTCAGTGGTACTCGCAACAGTTTCTCCTAGTGTTTGTGACACCCTTGGAAAGCACAAACATGGCGGGTGGAGAAAGAAAGACAAACATCAG

-525 CACGTTTAAAAAAGAATCTTCTgGgCGAAGAGCAAAGgCCGAGAATCAGGAGACAATTCTCCTTCAGgGGCTGCAGTAAAATTACTGATTAACCCAAAGCAAACTGATGTGT
ERE
Pit1
SRE

-413 TTACGGTCAAAATTAACCCAAACAGGTTGAAATATGAAAAGTTGTTTGAAATGTCTATAATTGACTGCAATTTGGTGATAAGAACCCAATTAAGCTGTTGTAGAAATGAATC NF1

-301 TAATAATTATAACCAAAAGGAATCATTCCAAAATCAGGCAGGGGGTGGGAGGTATTTGTATTTGGTACACTGGCAGAGCTGTTGTTITTCTAATCCTAGTCTGTGTTTGTAC NF1 LF-A1 ERE Pit1 Pit1 ERE

-189 TITCCTGTTTATTATGTCCACATTTGCAAGCAATAAAAGGGCATTATGTGCTGGTCATCCCATCTGCTTTTGAGATAAATCTATGTITGCATTICAAAGGGTCAAGGAACTC NF1. LF-A1 $\downarrow+1$

-77 TCCAGGGCAAACAAATTCTGGAGCCGCTGCTGCCAGATGGCGCGTATATTAAGTGGAAAGCGAGAAAAGCAATTTGCTGTGTTTCTGTTCCAGGGGAGAAGTCTCACCCCGA PRE

37 AAGACAGCAAAGAGgttagaaactaccgagaaattgtacaggcggttttcttctgttacatgtttctttctttgcatctaaaaagtttaggttcggagagaagctcagttc ttgttcaggcaaagctcttccaaaaaggtgtcaggaatatttaaccgaagaattgaaggttaagtttataacaccttttaagaattatgcacttctttatgtgggaggttcta gatttatctgtataactcactaatatgtagtctgtacttacagaaactctattctcgcagacaaaatggttttatcttgcattttgactgaactctacaaaagagacagaaa ERE acaattgatcagattattaggtacaaataagcgtgacctcaacaaaggcaagttatctgcataatttatccagctcaattgccaccttatactctgctattagcttgtcaatt ctgtaaacagaagcactgcaattaaatgggtaatttcccagcacacaaaagaactccgtaagtttcggagctgatacaatctcgccttcaaatctagtgtagcagtgggatgg ERE

gaaattcatatctgcatgagaaatttaaaaaccttttgttaaatactgaaaaccataacatatagccttcattcttcatatacccctgtattcttcataggtcaccagaaact ERE

gaaaatatgtagcagaagcattaagtgtttggacatgagcaaaggaaagggagaatgagtgacccaatatttatatgcgtacctctcttgagcatatttaattgtatatatat gtagcttttttacagcagcccttctttttactatcaggacttttcctacaaataaaggatatcagtaaagacttctctccgcacagGAAAGAAGGGACAACAATGCTGAGG 29PE

79 TTाGCCATCACCCTCTTGCTGTCATCACATCATCTACTGCCAGCAGTATGGATGTCTGGAAGgGGACACCCACAAAGCGAAGCCAAGTCCTGAGCCAAACATGCATGAATGC

192 ACTCTGTATTCTGAATgtaagagtggtttagtttttgctggag. . (0.82). . ccttactgtaagatctttgtttcattcctaacagCTTCCTGTTGCTATGCAAACTTCACA

234 GAGCAATTGGCTCATTCCCCAATAATTAAAGTAAGCAACAGCTACTGGAACAGATGTGGGCAGCTCAGTAAATCgtaagtcggctttgatgttgtgtgtgt. (1.1) . . ggct ttctcacccacaacagCTGTGAAGATTTCACAAAGAAAATCGAGTGCTTTTACCGGTGTTCTCCGCACGCTGCTCGCTGGATCGATCCCAGATATACTGCTGCTATTCAGTCT

405 GTTCCACTGTGTCAGAGCTTCTGTGATGACTGgtaggttcaaatcgctatcagaatatcttt. (3.0) . . ttccacactgatctccatgaagGTATGAAGCCTGCAAAGATGA

457 TTCCATTTGTGCTCATAACTGGCTGACGGACTGGGAACGGGATGAAAGTGGAGAAAACCACTGTAAGAGTAAATGCGTACCATACAGTGAGgtaagaaaacatggacagctgg ggcacaaaataaa. . (4.0) . gatgcgtgtttctcatttacacacagATGTATGCAAATGGGACCGACATGTGCCAGAGTATGTGGGGGGATCCTTTAAGGTGAGCGAATC 613 CTCCTGCCTCTGCTTGCAAATGAACAAGAAGgACATGGTGGCAATCAAGCACCTCCTCTCCGAAAGCTCAGAGGAAAGCTCCAGTATGAGCAGCAGTGAGGAGCACGCCTGCC

726 AAAAGAAACTCCTGAAGTTTGAGGCACTGCAGCAAGAGGAAGgGGAAGAGAGAAGATGAATTTTGGTGGATGAATATCAGGAGGAGAGGAATCATTGTGGAGGTTGTGCTCGG

839 GGCATCACAGCAGCCTGTCTTATCCCTCACTTCTGAGAACACAATAAATCAATGGTTGGCTATATT

Figure 1. Genomic organization and 5' flanking sequence of RCP gene. The RCP 5' flanking sequence and the exon regions are shown in upper case letters. Lower case letters represent the partial intron sequences. Numbers in parentheses indicate the sizes of the introns. Numbers on the left correspond to the nucleotide sequence shown in upper case. The sequence was subjected to Transfac database search (Prestridge 1991) and the results indicating the putative transcription factor binding sites are underlined and the respective factors are shown above the sequence. The putative transcription initiation site (TATA element) is shown in bold. The transcription start site is shown with an arrow and " +1 ". ATG codon and the polyadenylation site are shown as underlined, plain and wavy respectively. The oligonucleotide sequences (complimentary) used for primer extension are shown in italics and the regions corresponding to 29PE and 99PE are shown as overline and underline, respectively. The Genbank accession No. for this sequence is AF237863. 
of treatment and luciferase activities were assayed using the Dual Luciferase Kit (Promega Corpn., USA) in a luminometer (Turner designs, USA). The experiments were performed in triplicate with $n=2$ per treatment group for each construct. Data are plotted as 'fold over vehicle treated control measurements' for each deletion construct using the Graphpad Prizm software.

\section{Results}

\subsection{5 flanking region and genomic organization of RCP gene}

3.1a Isolation and characterization of $5^{\prime}$ flanking region: A chicken genomic library was screened using an RCP cDNA probe (Zheng et al 1988) and three clones, $\lambda$-CGR1, $\lambda$-CGR2 and $\lambda$-CGR3 were obtained after tertiary screening. These were found to be identical by restriction mapping, and hence $\lambda$-CGR2 was chosen for further analysis. EcoRI digestion of $\lambda$-CGR 2 resulted in an insert containing three products of $7 \mathrm{~kb}, 4 \mathrm{~kb}$ and $3 \mathrm{~kb}$ size. All three fragments hybridized to the RCP cDNA, indicating that they all contain exon regions of the RCP gene (data not shown). Oligonucleotides corresponding to the $5^{\prime}$ and $3^{\prime}$ sequences of RCP cDNA were hybridized to these fragments to identify their relative orientation with respect to each other. The $7 \mathrm{~kb}$ fragment was found to contain the $5^{\prime}$ region of the gene, while the 3 and $4 \mathrm{~kb}$ fragments contain the rest of the gene (data not shown). The $7 \mathrm{~kb}$ EcoRI fragment from $\lambda$-CGR2 was partially digested with Sau3A enzyme and the resulting overlapping fragments were subcloned into plasmid vector, pBS $\mathrm{KSII}^{+}$(Statagene cloning systems, Germany). One of the subclones has $1 \mathrm{~kb}$ of the $5^{\prime}$ flanking region along with a portion of the $5^{\prime}$ UTR. Sequence analysis of the $5^{\prime}$ flanking region using the Transfac database search (Prestridge 1991) revealed several putative transcription factor binding sites including six ERE half sites (figure 1).

3.1b Structure of RCP gene: The genomic organization of the chicken RCP gene was determined by sequence analysis and PCR amplifications of the various sub clones obtained from the inserts present in the RCP genomic $\lambda$-CGR2 clone. This analysis revealed five introns and six exons in the RCP gene (figure 1). All exon-intron junctions follow the consensus GT-AG rule. The first intron is present with in the $5^{\prime}$ untranslated region. Since introns in the $5^{\prime}$ UTR of several genes have been shown to have regulatory elements, the entire first intron of the RCP gene has been sequenced and analysed for transcription factor binding sites (Prestridge 1991). Sequence analysis revealed the presence of three estrogen response element (ERE) half sites and a PRE half site. The sizes of other introns were determined by PCR amplification using primers spanning the introns. The smallest intron was of $820 \mathrm{bp}$ and the largest was $4 \mathrm{~kb}$. The data are consistent with a previous report on the mapping of introns II and III that are involved in the splice site mutation of the chicken RCP gene (MacLachlan et al 1993).

\subsection{Mapping of transcriptional start site}

The transcriptional start site was mapped using the primer extension strategy as detailed $\S 2$. Hen liver total RNA was reverse transcribed using two specific and overlapping primers 29PE and 99PE whose extended products should differ by 26 nucleotides (figure 1). As shown in figure 2, lanes 1 and 4, primer extension by 99PE resulted in a product of 139 nucleotides and a 113nucleotide product with 29PE using total RNA from hen liver. This difference in the length of each extension product agrees with the size difference expected from these primers. Lanes 2 and 5 show extension by primers 99PE and 29PE using polyA ${ }^{+}$RNA from hen liver. However, due to very high lane background in these lanes,

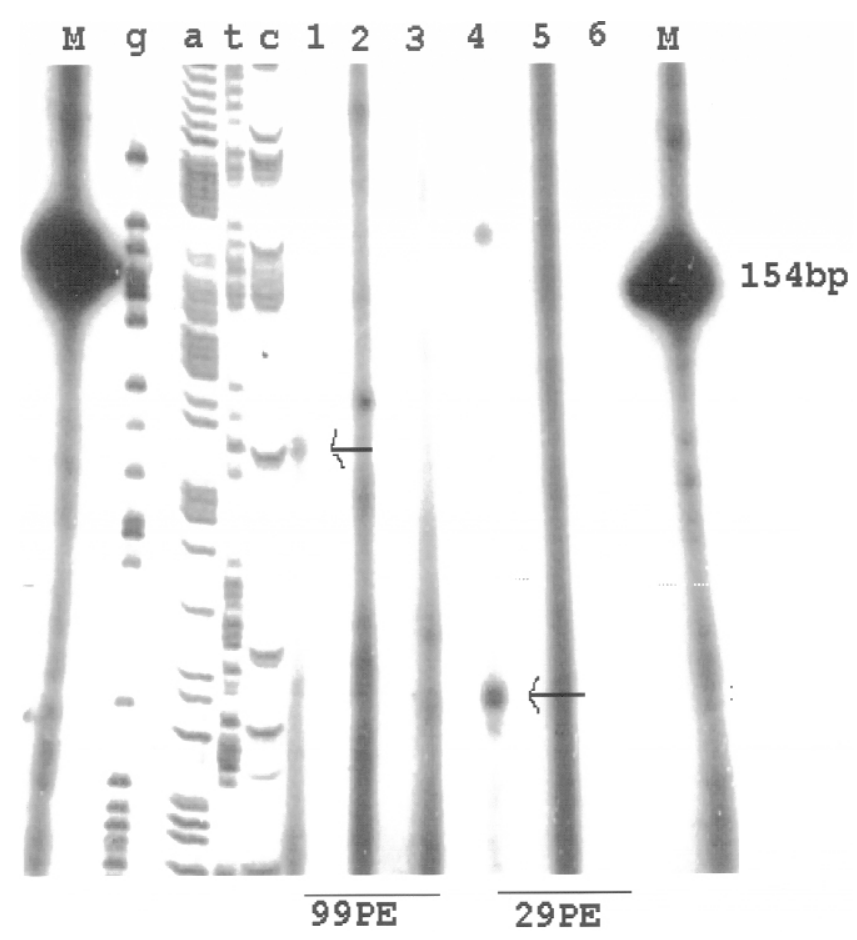

Figure 2. Transcription start site mapping by primer extension analysis. The primer extension on hen liver RNA was performed as described in $\S 2$ using two overlapping end labelled primers, 99PE and 29PE (figure 1). Arrows indicate the extended products. Lanes 1-3 using primer 99PE and 4-6 using 29PE. Lanes 1 and 4, $75 \mu \mathrm{g}$ of total RNA; lanes 2 and 5, $2 \mu \mathrm{g}$ polyA ${ }^{+} \mathrm{RNA}$; lanes 3 and $6,75 \mu \mathrm{g}$ of yeast tRNA. Lane M, end labelled pBR322 HinfI marker. g, a, t, c, Sequencing ladder using 99PE as the primer. 
they were difficult to interpret. Based on the size of the primer extension products using primers 99PE and 29PE, the transcription start site of the RCP gene is mapped at 68 nucleotides upstream of the ATG codon. It should be noted that primer 99PE has been used for both primer extension and generating the sequence ladder in figure 2. However, since there is an intron in the $5^{\prime}$ UTR (figure 1), the extension product that was generated by using this primer (which is in the second exon) can not correspond to the same nucleotide as in the sequence ladder. The sequencing template used in figure 2 is one of the genomic clones that contain the first intron. Hence, the sequencing reaction that was used in figure 2 was merely a sizing marker and refers to the size of extension product that was correlated to the sequence in figure 1 . The assignment of the start site is also consistent with the presence of a TATA element at -25 of the transcription start site mapped in this study.

\subsection{Regulation of RCP promoter by estrogen}

It has been well established that RCP protein is induced in immature pullets by estrogen (Murthy and Adiga 1978; DurgaKumari and Adiga 1986). In addition, data from our laboratory (Nandini Vasudevan and P Kondaiah, unpublished) indicated that RCP mRNA is also induced in the liver of male and female immature pullets after estrogen administration. Hence, in order to investigate the regulation of RCP $5^{\prime}$ flanking region by estrogen, a chicken hepatoma cell line, LMH2A (Leghorn Male Hepatoma) was used for transient transfection assays. LMH2A is a derivative from the parental LMH cell line, obtained by stable integration of a chicken estrogen receptor expression vector under the control of RSV promoter (Sensel et al 1994). LMH2A cell line was tested for RCP induction by moxesterol, a stable analogue of $17-\beta$ estradiol at a concentration reported for the induction of apoVLDL gene (Sensel et al 1994). Addition of moxesterol to LMH2A cells resulted in RCP mRNA induction at 6 and $12 \mathrm{~h}$ of treatment (figure 3 ).

The $5^{\prime}$ flanking region of RCP gene has six ERE half sites (figure 1). Hence, in order to test whether half siteEREs have any role in the regulation of this gene by estrogen, deletion constructs containing different number of ERE half sites from the chicken RCP $5^{\prime}$ flanking region were made in pGL3 basic vector and were designated as pRCPluc 1-4 (figure 4A). To test the promoter activity of the RCP deletion constructs in the presence of moxesterol, LMH2A cells were transiently transfected with pRCPluc 1-4 plasmids and luciferase activity was measured for each construct after moxesterol or vehicle treatment for $12 \mathrm{~h}$. The results shown in figure 4B indicate that all the $\mathrm{RCP}$ promoter deletion constructs respond to moxesterol after $12 \mathrm{~h}$ treatment. pRCP4 which contains all six half site EREs shows approximately 6-fold induction with moxesterol as compared with vehicle treated control. pRCPluc3 and pRCPluc2, both containing 3 half site EREs, show about 7- and 11-fold induction, respectively. The smallest construct, pRCPluc1, containing only the proximal two half site EREs, shows approximately 9-fold induction. This suggests that the region containing the proximal two half site EREs is sufficient to mediate estrogen induction of the chicken RCP gene and that the presence of a consensus palindromic ERE is not required for induction of RCP gene by estrogen.

\section{Discussion}

The molecular mechanisms of estrogen action have been elucidated by investigating induction by estrogen of the major egg yolk protein genes, vitellogenin (VTG) (Goldberger and Deeley 1980; Wolffe and Tata 1983) and the apo very low density lipoprotein (apo II), in the avian and amphibian liver (Wiskocil et al 1980) as well as egg white protein genes such as ovalbumin in the avian oviduct (O'Malley et al 1979). Vitamin carrier proteins such as RCP are minor egg yolk and egg white constituents. Among the hitherto characterized proteins, RCP is the only protein other than conalbumin that is synthesized in both liver and oviduct under estrogenic stimulus. However, conalbumin expression in the liver is constitutive and upregulated only 2-fold by estrogen (Lee et al 1978) whereas RCP induction requires the presence of estrogen. Hence, RCP gene is a good model to investigate the mechanisms of estrogen induction common to both liver and oviduct.

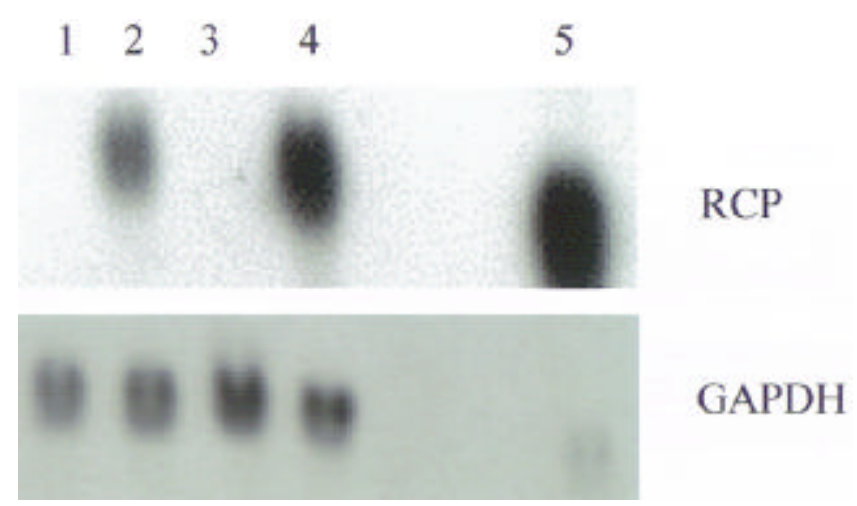

Figure 3. Northern blot analysis of RCP induction in LMH2A cell line by moxesterol. $40 \mu \mathrm{g}$ of total RNA from LMH2A cells was analysed on a $1.2 \%$ agarose formaldehyde gel, transferred onto nylon membrane and hybridized to RCP cDNA probe. Chicken GAPDH was used as an internal control to normalize the amount of RNA loaded. Lanes 2 and 4, after 6 and $12 \mathrm{~h}$ of moxesterol treatment; lanes 1 and 3, vehicle treated cells; lane 5, hen liver RNA used as a positive control. 
Molecular characterization of the RCP gene structure reveals five introns, including an intron of $864 \mathrm{bp}$ in the $5^{\prime}$ untranslated region. Three potential ERE half sites are present in the first intron. The significance of these hormone regulatory elements in the first intron is not yet established. However, in several genes, the introns present in the $5^{\prime}$ UTR were found to contain functional regulatory elements. For example, in the apo II gene, the inclusion of the first intron in the promoter constructs results in a 4-fold enhancement of promoter driven reporter gene expression (Berkowitz and Evans 1992).
Transcription start site mapping of RCP gene by primer extension analysis shows the +1 nucleotide to be 68 nucleotides upstream of the translation start. The sequence of the $5^{\prime}$ flanking region shows a number of transcription factor binding sites as revealed by a 'Transfac' database search (Prestridge 1991) (figure 1). Prominent among these are six half site EREs which are perfect matches with either of the two consensus half sites which comprise the ERE palindrome. There is appparently no consensus palindromic ERE in this sequence. The three proximal EREs in the chicken RCP promoter match the first half

(A)
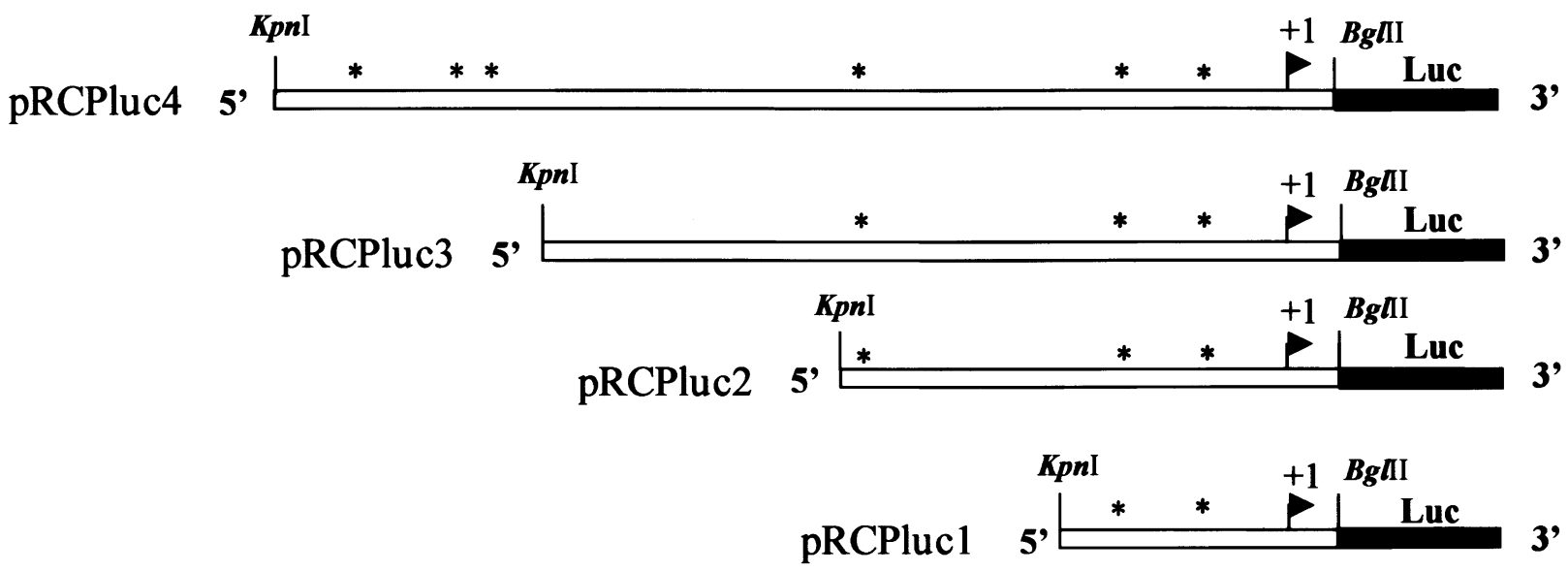

(B)

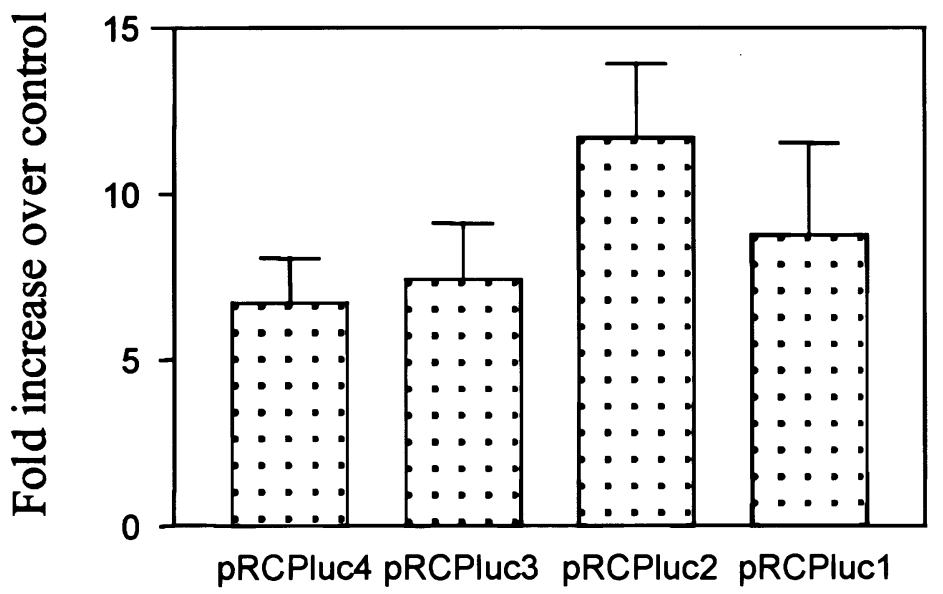

Figure 4. Promoter activity of RCP after moxesterol treatment. (A) Schematic of the promoter regions placed upstream of the luciferase in pGL3 basic vector. Asterisks indicate the approximate position of ERE half sites in the promoter sequence. The transcription start site is shown as +1 and the restriction enzyme sites used for cloning of the respective promoter regions in pGL3 basic vector are depicted above the sequence. The regions of the RCP gene (figure 1) cloned in pGL3 basic vector are from -216 to +50 (pRCPluc1), -437 to +50 (pRCPluc2), -697 to +50 (pRCPluc3) and -973-+ 50 (pRCPluc4). (B) Results of transient transfection experiments using the promoter deletion constructs pRCPluc 1-4, after moxesterol treatment depicted as fold increase over the respective vehicle treated controls. The error bars represent mean \pm SEM. 
site of the ERE palindrome while the distal EREs are perfect half sites of the second half of the ERE palindrome. The progressive deletion constructs which contain varying numbers of ERE half sites have been tested for estrogen induction in a homologous chicken hepatoma cell line. The promoter region containing the proximal two ERE half sites is sufficient to confer estrogen inducibility on the RCP promoter. Inclusion of promoter regions containing more consensus ERE half sites does not show either additive or synergistic induction by estrogen.

Hamajima et al (1999) have reported an estrogenmediated enhancer activitiy of different regions of a $2.5 \mathrm{~kb}$ chicken RCP $5^{\prime}$ flanking sequence. They also indicated a transcription start site that is 5 nucleotides upstream of the start site mapped in this study. However, in the absence of mapping data in that report, it is difficult to interpret this discrepancy. The $5^{\prime}$-most $1.5 \mathrm{~kb}$ region contains an imperfect ERE and the rest of the $1.0 \mathrm{~kb}$ region had 6 ERE half sites. The sequence reported in this report is in agreement with the corresponding region reported by Hamajima et al (1999). In an enhancer activity assay using a SV-40 basal promoter/ reporter vector and MCF 7 human breast carcinoma cell line, a weak induction (3fold) in response to estrogen has been demonstrated for the distal $1.5 \mathrm{~kb}$ promoter region that contains an imperfect ERE. In addition, the $1.0 \mathrm{~kb}$ region containing ERE half sites also showed estrogen responsiveness in the same assay. Surprisingly, there was no synergism between the region containing the imperfect ERE and the $1 \mathrm{~kb}$ region which contains the six ERE half sites. This is in contrast to the known mechanism of estrogen regulated gene expression wherein in the ovalbumin gene, addition of more EREs results in an enhanced induction by estrogen (Kato et al 1992). In addition, estrogen mediated enhancer activity of the first intron in the $5^{\prime}$ UTR of RCP gene was also demonstrated by the same strategy. However, Hamajima et al (1999) did not use the RCP basal promoter and a liver specific cell line in their study. In this report, we have characterized the estrogen regulatability of the RCP promoter region using its own basal promoter in a homologous cell line. We have also addressed the question whether the region containing all the six half sites is required for estrogen induction of the promoter. We have demonstrated that the region containing only two of the six ERE half sites is sufficient to confer estrogen inducibility to this gene. It remains to be demonstrated whether the enhancer activity of the $5^{\prime}$-most region containing the imperfect ERE and the first intron confers estrogen inducibility to the RCP promoter.

The RCP promoter also contains binding sites for NF1, Pit1, Oct1, AP3 and most importantly, a hepatocyte specific factor, LF-A1. The presence of the liver specific transcription factor LF-A1 has been shown to play a role in the liver specific expression of several genes (Beekman et al 1991; Citarella et al 1993). LF-A1 sites, present in the proximal region of the chicken RCP promoter, may confer tissue specificity in the hepatic expression of this gene. It is possible that the presence of tissue specific transcriptional activators may play a role in the estrogenmediated induction of the RCP gene in both the liver and the oviduct. Further analysis of the promoter will provide clues for the molecular mechanisms involved in the tissue restricted estrogen regulation of the chicken RCP gene.

\section{Acknowledgements}

The authors wish to thank Prof. Pierre Chambon and Prof. Harold White for the gifts of the chicken genomic library and the RCP cDNA respectively. We are grateful to Prof. $\mathrm{P} R$ Adiga for his support and encouragement and Dr David Williams for his advice on the culture of LMH cell lines. NV is recipient of a fellowship from the Council for Scientific and Industrial Research, New Delhi. The work was supported by the Department of Science and Technology, New Delhi by a grant to PK.

\section{References}

Adiga P R 1994 Riboflavin Carrier Protein in reproduction; in Vitamins as ligands in cell communication (ed.) K Dakshinamurti (Cambridge: Cambridge Univ. Press) pp 136-176

Ausubel F, Brent R, Kingston R E, Moore D D, Seidman J G, Smith J A and Struhl K 1995 Current protocols in molecular biology (New York: John Wiley)

Beekman J M, Wijnholds J, Schippers I J, Pot W, Gruber M and Geert A B 1991 Regulatory elements and DNA Binding proteins mediating transcription from the chicken very low density apolipoprotein II gene; Nucleic Acids Res. 19 53715377

Berkowitz E A and Evans M I 1992 Functional analysis of regulatory regions upstream and in the first intron of the estrogen responsive chicken very low density apolipoprotein II gene; J. Biol. Chem. 267 7134-7138

Chomczynski P and Sacchi N 1987 Single step method of RNA isolation by Acid guanidinium thiocyanate phenol chloroform extraction; Anal. Biochem. 162 156-159

Church G M and Gilbert W 1984 Genomic Sequencing; Proc. Natl. Acad. Sci. USA 81 1991-1995

Citarella F, Misiti S, Felici A, Aiuti A, La Porta C and Fantoni A 1993 The $5^{\prime}$ sequence of human factor XII gene contains transcription regulatory elements typical of liver specific, estrogen-modulated genes; Biochim. Biophys. Acta 1172 197-199

DurgaKumari B and Adiga P R 1986 Hormonal induction of riboflavin carrier protein in the chicken oviduct and liver: a comparison of kinetics and modulation; Mol. Cell Endocrinol. 44 285-292

Goldberger R F and Deeley R G 1980 Gene regulation by steroid hormones (eds) A K Roy and J H Clark (New York: Springer-Verlag) pp 32-56 
Hamajima S and Ono S 1995 Sequence of a cDNA encoding turtle riboflavin binding protein: a comparison with avian riboflavin binding protein; Gene 164 279-282

Hamajima S, Hirano H, Horiuchi S and Ono S 1999 5'-Upstream structure of the gene coding for chicken riboflavin-binding protein and its relation to estrogen induction; IUBMB Life $\mathbf{4 8}$ 293-298

Hamazume Y, Mega T and Ikenaka T 1984 Characterisation of hen egg white and yolk riboflavin binding proteins and amino acid sequence of egg white riboflavin binding protein; J. Biochem. 95 1633-1644

Hamazume Y, Mega T and Ikenaka T 1987 Position of disulfide bonds in riboflavin binding protein of hen egg white; J. Biochem. 101 217-223

Kato S, Tora L, Yamauchi J, Masushige S, Bellard M and Chambon P 1992 A far upstream estrogen response element of the ovalbumin gene contains several half palindromic 5' TGACC 3' motifs acting synergistically; Cell 68 731742

Lee D C, McKnight S and Palmiter R D 1978 The action of estrogen and progesterone on the expression of the transferrin gene; J. Biol. Chem. 253 3494-3523

MacLachlan I, Nimpf J, White H B and Schneider W J 1993 Riboflavinuria in the rd chicken: 5' splice site mutation in the gene for riboflavin binding protein; J. Biol. Chem. 268 23222-23226

Murthy U S and Adiga P R 1978 Estrogen induced synthesis of riboflavin binding protein in immature chicks: kinetics and hormonal specificity; Biochim. Biophys. Acta. 538 364-375
O’Malley B W, Roop D R, Lai E C, Nordstrom J L, Catterall J T, Swaneck G E, Colbert D A, Tsai M J, Duggiczyk A and Woo S L C 1979 The ovalbumin gene: organization, structure, transcription and regulation; Rec. Prog. Horm. Res. 35 $1-46$

Prestridge D S 1991 SIGNAL SCAN: A computer program that scans DNA sequences for eukaryotic transcriptional elements; Cabios 7203

Sambrook J, Fritsch D R and Maniatis T 1989 Molecular Cloning: A laboratory manual, 2nd edition (New York: Cold Spring Harbor Laboratory Press)

Sensel M G, Binder R, Lazier C B and Williams D L 1994 Reactivation of apolipoprotein II gene transcription by cycloheximide reveals two steps in the deactivation of estrogen receptor mediated transcription; Mol. Cell Biol. 14 1733-1742

White H B and Merrill A H 1988 Riboflavin carrier proteins; Annu. Rev. Nutr. 8 279-299

Wiskocil R, Bensky R, Dower W, Goldberger R F, Gordon J I and Deeley R G 1980 Coordinate regulation of two estrogen dependent genes in avian liver; Proc. Natl. Acad. Sci. USA 77 $4474-4478$

Wolffe A P and Tata J R 1983 Coordinate and noncoordinate estrogen induced expression of A and B groups of vitellogenin genes in male and female Xenopus hepatocytes; Eur. J. Biochem. 130 365-372

Zheng D B, Lim H M, Pene J and White H B 1988 Chicken riboflavin binding protein: cDNA sequence and homology with milk folate binding protein; J. Biol. Chem. $26311126-$ 11129

MS received 21 November 2000; accepted 25 January 2001

Corresponding editor: VIDYANAND NANJUNDIAH 